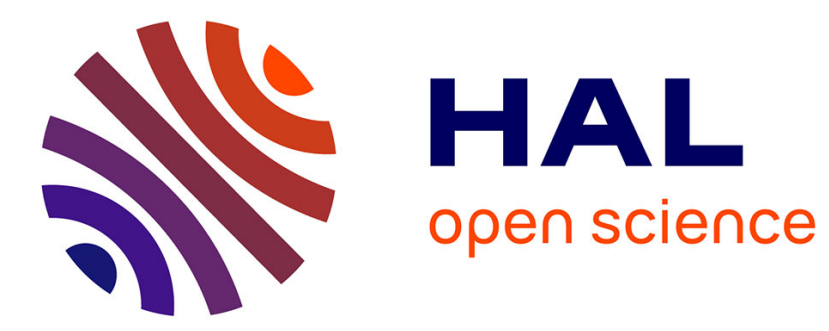

\title{
Les "patrons" français du secteur de l'habillement. Pourquoi ont-ils délocalisé au Maroc?
}

Brahim Labari

\section{To cite this version:}

Brahim Labari. Les "patrons" français du secteur de l'habillement. Pourquoi ont-ils délocalisé au Maroc?. Hommes \& migrations, 2007, 1266, pp.108 - 124. 10.3406/homig.2007.4591 . hal-03240155

\section{HAL Id: hal-03240155 \\ https://hal.univ-lorraine.fr/hal-03240155}

Submitted on 28 May 2021

HAL is a multi-disciplinary open access archive for the deposit and dissemination of scientific research documents, whether they are published or not. The documents may come from teaching and research institutions in France or abroad, or from public or private research centers.
L'archive ouverte pluridisciplinaire HAL, est destinée au dépôt et à la diffusion de documents scientifiques de niveau recherche, publiés ou non, émanant des établissements d'enseignement et de recherche français ou étrangers, des laboratoires publics ou privés.

\section{(ㅇ)(1) $\$$}

Distributed under a Creative Commons Attribution - NonCommercial - NoDerivatives| 4.0 


\section{Les "patrons" français du secteur de l'habillement. Pourquoi ont-ils délocalisé au Maroc}

\section{Brahim Labari}

\section{Résumé}

Dans une perspective sociohistorique, l'auteur met l'accent sur le poids des liens et des représentations historiques, liées à l'imaginaire colonial, qui expliquent les raisons de ces délocalisations et les stratégies que ces patrons français mobilisent pour tenter de réussir leur intégration à la société marocaine. Il distingue deux types de patrons : les "néocoloniaux prudents" et les "conquérants aventuriers" qui, dans un contexte de mondialisation, attachent moins d'importance aux préjugés qu'aux avantages que leur procure leur statut social.

\section{Citer ce document / Cite this document :}

Labari Brahim. Les "patrons" français du secteur de l'habillement. Pourquoi ont-ils délocalisé au Maroc. In: Hommes et Migrations, n¹266, Mars-avril 2007. Nouvelles figures de l'immigration en France et en Méditerranée. pp. 108-124; doi : https://doi.org/10.3406/homig.2007.4591

https://www.persee.fr/doc/homig_1142-852x_2007_num_1266_1_4591

Fichier pdf généré le 08/05/2018 


\section{Les "patrons" français \\ du secteur de l'habillement}

Pourquoi ont-ils délocalisé au Maroc ?

Par Brahim Labari,

Enseignant-chercheur, Laboratoire CNRS "Genre, travail et mobilité", université Paris X-Nanterre

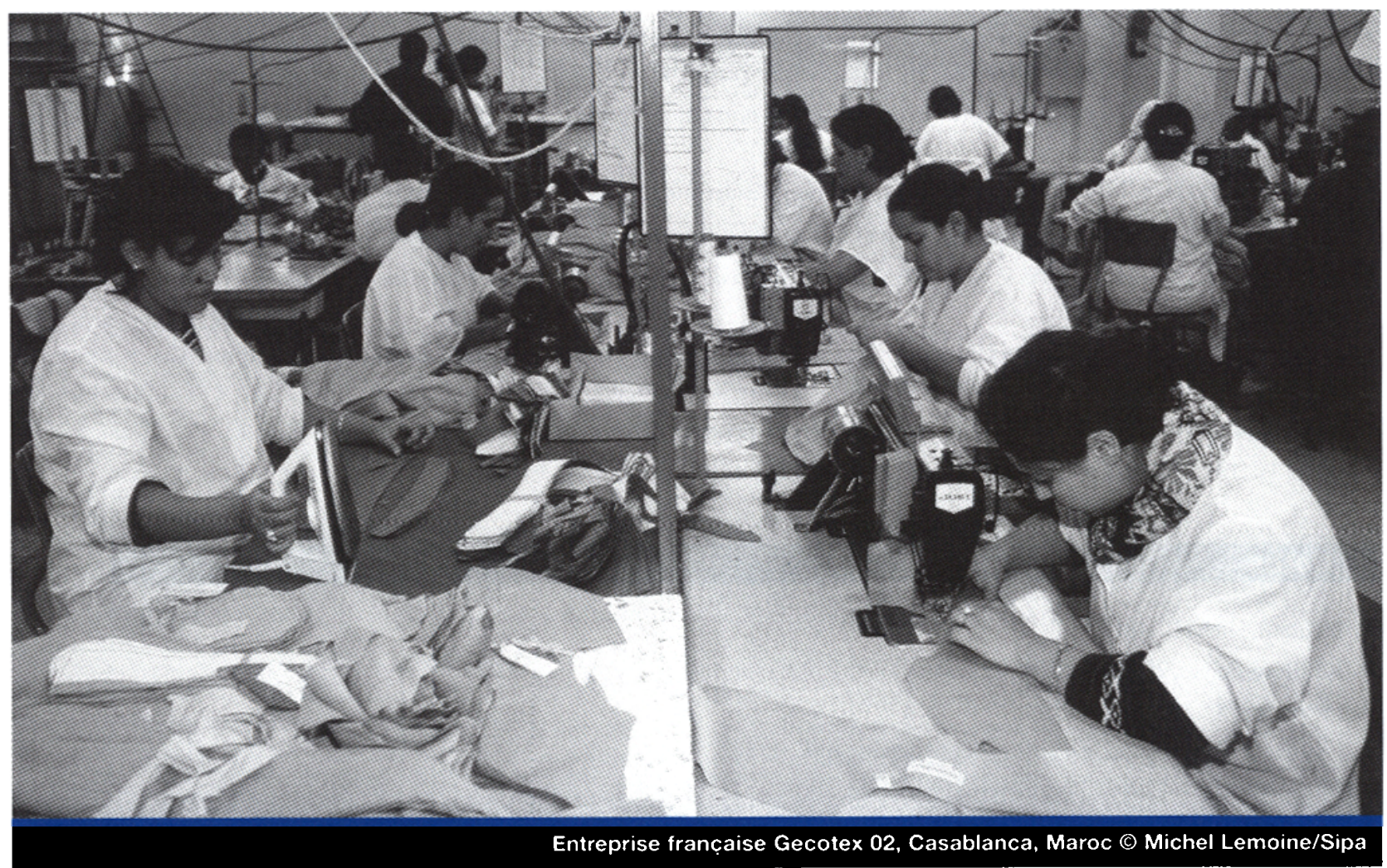

Dans une perspective sociohistorique, l'auteur met l'accent sur le poids des liens et des représentations historiques, liées à l'imaginaire colonial, qui expliquent les raisons de ces délocalisations et les stratégies que ces patrons français mobilisent

pour tenter de réussir leur intégration à la société marocaine. II distingue deux types de patrons : les "néocoloniaux prudents" et les "conquérants aventuriers" qui, dans un contexte de mondialisation, attachent moins d'importance aux préjugés qu'aux avantages que leur procure leur statut social. 
À partir d'un échantillon de cinquante "patrons" français ayant délocalisé leur entreprise au Maroc, cet article ${ }^{\text {I1 }}$ entend explorer les raisons empiriques de la délocalisation. À l'aune d'une distinction entre l'économique et le culturel, il s'agit de tenter d'établir une typologie de ces patrons, "néocoloniaux prudents" et "conquérants aventuriers", en considérant la dynamique historique des rapports franco-marocains. La délocalisation est entendue comme une rupture avec une source nationale d'approvisionnement, de production ou de transformation, la France, au profit d'une source extérieure, en l'occurrence le Maroc. Le choix du terme de "patron" requiert une justification méthodologique. Les propos tenus par les intéressés mettent en évidence la prévalence de deux auto-désignations : "patron" et "investisseur". Cette dernière paraissant avoir une connotation essentiellement économique, la première, plus pertinente, embrasse non seulement une vision économique mais également une dimension sociétale, faite d'implications dans les rapports sociaux en contexte marocain.

Comment caractériser les patrons français du secteur de l'habillement délocalisant leurs entreprises au Maroc? À la lumière d'une enquête menée par questionnaire et entretiens auprès d'un échantillon de cinquante patrons français établis à Casablanca, il s'agit d'abord de mener un travail de définition et d'identification : quelles sont les caractéristiques sociologiques de ces patrons? Quels projets de délocalisation mènent-ils? Quelle cst la part de l'économique et du culturel dans leurs projets? Comment peut-on caractériser leurs contacts avec l'environnement marocain? Nous tenterons ensuite d'esquisser une typologie des patrons enquêtés, en fonction de leurs motivations à délocaliser et de leur perception du contexte sociétal marocain. Nous soulignerons l'importance des facteurs historiques qui rentrent en compte dans ces choix, la continuité des représentations sociales, héritées de la période coloniale, qui déterminent encore le rapport qu'entretiennent certains patrons français avec le Maroc et les Marocains.

\section{Qui sont les "patrons" qui délocalisent ?}

Une lecture sociologique des caractéristiques permet de mettre en évidence à la fois les raisons de la délocalisation et les stratégies de légitimation mobilisées en vue de l'intégration dans la société locale ${ }^{\prime 2}$.

Globalement, la population de l'échantillon est relativement âgée et exclusivement masculine. Cette structure par âge conforte l'hypothèse de l'existence d'affinités historiques avec le Maroc. Sur les 50 patrons, 33 ont déclaré avoir un lien préalable avec le Maroc. La plupart ont déjà connu le Maroc, soit au cours d'un voyage "exploratoire", 
soit par l'intermédiaire d'une connaissance ou d'une famille déjà installée sur place. La variable de l'âge nous renseigne sur l'intensité du lien, mais également sur le vécu de différents événements ayant trait aux rapports franco-marocains : "J'arrive à Anfa, le quartier d'une certaine France, l'architecture porte encore les traces d'un certain Prost. Je me sentais chez moi, tellement le souvenir de cette période rejaillissait en moi quand, gosse,

\section{Tableau I}

\begin{tabular}{|l|c|}
\hline Tranches d'âge & $\begin{array}{c}\text { Effectifs } \\
\text { (Pourcentage) }\end{array}$ \\
\hline $30-40$ ans & $9(18)$ \\
\hline $40-50$ ans & $11(22)$ \\
\hline $50-60$ ans & $23(46)$ \\
\hline $60-65$ ans & $7(14)$ \\
\hline Total & $50(100)$ \\
\hline
\end{tabular}

\section{Tableau II}

\begin{tabular}{|c|c|}
\hline $\begin{array}{l}\text { Situation } \\
\text { matrimoniale }\end{array}$ & $\begin{array}{l}\text { Effectifs } \\
\text { (Pourcentage) }\end{array}$ \\
\hline Célibataire & $5(10)$ \\
\hline $\begin{array}{l}\text { Marié ou vivant } \\
\text { en concubinage }\end{array}$ & $29(58)$ \\
\hline Divorcé & $16(32)$ \\
\hline Total & $50(100)$ \\
\hline
\end{tabular}
mes parents à l'époque étaient fonctionnaires, je fréquentais la crèche missionnaire, et là un tas de bons moments défilait dans ma tête, à l'investissement affectif, je me suis décidé à implanter mon unité dans cette ville".

Plus de $60 \%$ des patrons enquêtés ont commencé leur carrière bien après l'indépendance du Maroc (1956), dans la continuation d'une affaire familiale appelée à se succéder à elle-même dans un environnement devenu rude. Leur délocalisation les place face à un choix : "Partir ou mourir";"Mon capital, mes machines et mon carnet d'adresses ou la fermeture et la reconversion". Le choix de délocaliser est retenu, favorisé par la perception d'un marché marocain ouvert au capital étranger.

Les patrons mènent une vie de couple, parfois de couple mixte. 11 d'entre eux ont contracté un mariage avec une Marocaine (38\% des mariés). Le choix du Maroc relève le plus souvent du désir d'y rester pour une longue période, voire pour la vie. Les 60-65 ans sont les plus convaincus de finir leur carrière au Maroc. Ils préferent "une jeune société où tout est encore possible malgré les difficultés inhérentes à l'implantation". La critique de la société française est souvent sévère : la "désindustrialisation", qu'ils imputent aux politiques suivies; la "culture soixante-huitarde", issue d'une "hystérie collective". Le refus de dépendre d'un maître est selon eux à l'origine du mal français. Pour des raisons d'ordre affectif et familial, les "expatriés" ont choisi de s'installer définitivement au Maroc. L'effet de génération tient lieu dans leur cas de continuité. Les retraités ambitionnent de continuer leurs affaires. Ils se font appeler "P-D.G." alors que les plus jeunes se font désigner par le terme de "manager". Il y a dans ces appellations une différence de style. 
Les célibataires sont l'archétype du nomade, et considèrent Casablanca comme leur "résidence secondaire". Plus jeunes et plus ambitieux, ils ont majoritairement entre 30 et 40 ans. Ils font des allers-retours entre la France et le Maroc, mais ne connaissent pas de Jus Soli. Ils s'adaptent au pays.

Les patrons originaires du pourtour français de la Méditerranée sont plus nombreux à délocaliser, en raison des réseaux historiques existants dans cette région. Les prémisses d'un métissage méditerranéen existent bien avant le $\mathrm{XIX}^{\mathrm{e}}$ siècle ${ }^{i 3} ;$ des réseaux économiques se sont développés pendant le protectorat et se sont poursuivis après l'indépendance. L'ouverture du Maroc à son bassin méditerranéen est favorisée par son double littoral, méditerranéen et atlantique, et par l'influence des différentes conquêtes dont il a fait l'objet ${ }^{(4)}$. Les actions politiques et économiques régionales revêtent une grande importance dans l'incitation des entreprises à développer un partenariat avec la rive sud de la Méditerranée, la proximité géographique aidant. Les villes à dimension méditerranéenne comme Marseille sont de ce point de vue légions. Près du tiers des patrons sont originaires de la région PACA

Tableau III

\begin{tabular}{|l|c|}
\hline $\begin{array}{l}\text { Région de l'unité } \\
\text { délocalisée }\end{array}$ & $\begin{array}{c}\text { Effectifs } \\
\text { (Pourcentage) }\end{array}$ \\
\hline Région parisienne & $8(16)$ \\
\hline Nord-Pas-de-calais & $9(18)$ \\
\hline Rhône-Alpes & $14(28)$ \\
\hline $\begin{array}{l}\text { Provence-Alpes- } \\
\text { Côte d'Azur } \\
\text { Total }\end{array}$ & $19(38)$ \\
\hline
\end{tabular}
(Provence-Alpes-Côte d'Azur). Les actions impulsées par son conseil régional sont conçues pour encourager le partenariat économique entre les deux rives de la Méditerranée. Plusieurs chartes ont ainsi été élaborées dans ce sens. Un élément récurrent est mis en valeur par la plupart des collectivités territoriales : la Méditerranée est le socle commun à l'État et aux collectivités locales. Les régions à tradition industrielle comme Rhône-Alpes sont également concernées par le phénomène. La région Nord est représentée également en raison de l'importance de son secteur textile, dont la crise, pendant les années soixante-dix, a sans doute joué un rôle décisif dans la délocalisation. Les délocalisations proviennent à un degré moindre de la région parisienne.

\section{Les origines empiriques de la "délocalisabilité"}

En règle générale, la littérature économique fonde les raisons de la délocalisation sur la théorie des avantages comparatifs de D. Ricardo. Cette théorie postule que chaque pays développe une spécialisation dans un secteur d'activité 
susceptible de le doter d'un avantage. Mais, si le critère économique est central dans le choix d'un pays, voire d'une région, d'autres modes de rationalité peuvent être recherchés en interrogeant les subjectivités et les expériences des patrons qui délocalisent.

La délocalisabilité, terme heuristique, permet de mettre en lumière, du point de vue des patrons français, les atouts et obstacles du Maroc comme terre de délocalisation. Elle se rapporte à la faisabilité d'une délocalisation. Certes, le Maroc n'est pas programmé pour accueillir des entreprises françaises ; mais il existe une prédisposition de ce pays à répondre favorablement à des stratégies de délocalisation. Le terme de "délocalisabilité" comprend la prospection des facteurs facilitant une meilleure réception du projet de délocalisation et sa rentabilité économique future.

Trois questions ont été posées: "En amont, pourquoi le choix du Maroc et la ville de Casablanca?"; "En aval, quels sont les atouts et les obstacles rencontrés au cours de l'implantation?"; "Comment s'est construite la légitimation sociale et culturelle de cette opération économique?”

\section{Tableau IV}

\begin{tabular}{|l|c|}
\hline Raisons de la délocalisation & Pourcentage (effectifs) \\
\hline L'usage de la langue française & $29(37)$ \\
\hline La proximité géographique & $16(21)$ \\
\hline La bonne implantation du secteur textile-habillement & $7(12)$ \\
\hline La main-d'ceuvre moins chère & $10(15)$ \\
\hline Autres avantages économiques & $7(09)$ \\
\hline Existence d'une communauté française & $5(07)$ \\
\hline Possibilité de s'associer à un entrepreneur local & $13(17)$ \\
\hline Divers : avantages de service(5) ; stabilité politique.... & $9(13)$ \\
\hline Total
\end{tabular}

De manière anthropologique, nous analysons le rapport qu'entretiennent les patrons avec la culture marocaine, perçue et vécue dans l'approche du terrain local. En règle générale, il ressort que le Maroc comporte toutes les vertus d'un pays accueillant et tolérant. Le plus souvent, c'est l'authenticité des Marocains et leur hospitalité qui sont louées. En revanche, l'administration est critiquée pour sa lenteur et son "imprévisibilité".

Parmi les facteurs ayant justifié la décision de délocaliser, figurent en bonne place la langue, la proximité géographique et la connaissance du milieu local marocain. 


\section{L'atout de la langue française et la proximité géographique}

Au lendemain de l'indépendance nationale, l'une des questions cruciales qui se posent au Maroc fraîchement indépendant est de savoir s'il faut promouvoir la langue arabe (langue de la lutte et de la contestation) ou reconduire le français (la langue du colonisateur). Sans trancher, le Maroc maintient les dcux langues, aussi bien dans le cadre de l'enseignement que dans les administrations. Le français continue à être enseigné à partir de la $3^{\mathrm{c}}$ année du cycle primaire, et les documents administratifs comportent les deux langues. Du point de vue des patrons français, la langue, moyen de communication, permet de faciliter le contact avec les Marocains et l'administration. Si la langue française est surtout l'apanage des milieux sociaux intellectuels ou aisés, elle est valorisée par l'usage et par sa portée symbolique. Par l'usage du français, les patrons sont dispensés d'apprendre l'arabe ou le berbère. Les autres communautés, à l'exception de la communauté de l'enclave espagnole dans le nord, doivent faire l'effort d'apprendre le français ou l'arabe. C'est sans nul doute un avantage par rapport à d'autres communautés linguistique (anglaise, américaine ou espagnole): "Un gendarme t'arrête pour un excès de vitesse, tu lui fais un sourire et tu lui parles en français, il te répond et il est tout content, et l'affaire est réglée", dit un des patrons interrogés.

La langue du milieu d'affaires reste le français, de même que pour l'élite politique, à commencer par le roi - le roi actuel comme son père ayant fait leurs études en France. C'est une fenêtre sur l'extérieur et l'outil de communication en vigueur dans les secteurs de prises de décision. Les deux télévisions marocaines émettent en arabe et en français, de même que la radio du Maghreb (Médi 1). La consécration de la langue française est une donnée constante dans le discours politique. Même les partis les plus nationalistes ne contestent pas sa place dans la stratégie d'ouverture du Maroc aux nouveaux moyens de communication.

Par rapport à l'Europe, le Maroc est géographiquement bien situé pour les patrons français. Cette position géographique ferait de lui un trait d'union entre l'Afrique et l'Europe, entre le monde musulman et chrétien. Cette proximité avec l'Europe et son enracinement en terre africaine rappellent la rhétorique de l'arbre, utilisée par le discours politique ${ }^{\prime 6}$ pour tirer profit de cette position géographique. La proximité physique du marché européen favorise le choix du Maroc. Elle offre surtout un avantage comparatif s'agissant du coût de transport des marchandises. La location d'un camion, transport le plus utilisé pour acheminer des commandes moyennes, coûte approximativement 6000 dirhams, soit 600 euros. Pour les grosses commandes et pour les convois exceptionnels, la location d'un bateau, qui part du port de Casablanca vers la ville de Sète, est plus avantageuse. 


\section{Textile/habillement : qualité de la production, main-d'œuvre bon marché}

Les patrons louent la tradition de ce secteur au Maroc en mettant l'accent sur l'image de la qualité de la production marocaine auprès de la clientèle du secteur. Les statistiques vont dans le même sens : l'accroissement des exportations d'une part, le développement des investissements de l'autre, sont des indices qui confortent la certitude du choix du Maroc. En effet, à partir de 1987, les restructurations du secteur européen provoquent la délocalisation de nombreuses entreprises de l'Hexagone. Pendant cette période, les investissements étrangers représentent en moyenne $30 \%$ des investissements textiles du Maroc. On assiste également à une croissance accélérée des exportations, particulièrement dans la confection et la bonneterie, et à la création et l'expansion d'unités spécialisées dans les branches exportatrices. Un produit qui s'exporte bien attire plus que les autres. Le taux de couverture des importations par les exportations est passé de $130 \%$ en 1986 à $166 \%$ en 1991.

Une attitude "protectrice" prédomine chez bon nombre de ces patrons : outre le motif économique, ils expriment leur désir de porter le secteur de l'habillement face à la concurrence, mais aussi de hisser le Marocain au rang de travailleur rigoureux "ne s'en remettant plus au ciel pour son salut". La main-d'ceuvre marocaine présente des avantages. Son accroissement continu (les jeunes représentent plus de $50 \%$ de la population marocaine selon le dernier recensement), la disponibilité sur le plan local d'une main-d'œuvre féminine en renouvellement constant sont autant de facteurs qui favorisent la délocalisation. Marcel Albert, patron d'Albert SA, rappelle laconiquement la différence de salaires entre ouvriers de différentes nationalités: "Avec le salaire d'une ouvrière française, vous pouvez employer, à temps de travail et productivité équivalents, 9 Marocains, 25 Thaillandais, 35 Chinois, 65 Russes ou 70 Vietnamiens."

Plusieurs patrons évoquent aussi l'exemplarité du travail des immigrés marocains qu'ils ont déjà employés en France et mettent en avant les expériences communes : "Les Marocains travaillent sans chicaner, leur réputation est bien établie. Les patrons de l'automobile en savent quelque chose en France. L'Algérien, par le passé douloureux des relations entre la France et l'Algérie, est réfractaire à toute hiérarchie, s'agissant surtout d'une hiérarchie française". La main-d'ceuvre marocaine est considérée comme docile. Les autres avantages économiques à la dćlocalisation se rapportent aux effets de l'abrogation de la loi dite de la marocanisation, jetant les bases d'un cadre juridique propice au capital étranger. Ce cadre inscrit aussi l'économie marocaine dans le libre-échange et l'économie de marché. Il offre aux investisseurs étrangers, y com- 
pris pour les petits patrons français du textile-habillement, une série d'avantages fiscaux. Parmi ces facilités, on peut citer : exonération du droit d'importation sur les matériels, outillages en biens d'équipement ; exonération de la TVA (taxe sur la valeur ajoutée) sur le matériel importé ou acquis localement ; exonération de l'IS (impôt sur les sociétés) pendant les cinq premières années d'exercice.

Tous ces éléments ont porté la France au premier rang des investissements directs, de commerce, d'aide publique et de coopération scientifique et culturelle. Elle compte en effet 500 sociétés au Maroc, avec une place pionnière dans tous les secteurs stratégiques : les télécommunications avec Vivendi Universal, la construction avec Lafarge, la distribution avec Auchan, l'eau et l'électricité avec la Lyonnaise des Eaux, devenue Lydec, le tourisme avec le Club Méditerranée. Pour le secteur du textile-habillement, plusieurs groupes sont représentés au Maroc (Gaulliez, Billon, Sotap Carol, Chantelle...)

Lorsque le Maroc accède à l'indépendance, les Français qui y restent constituent un contingent limité, chargé à la fois de former les Marocains et d'intégrer les services "Avec le salaire d'une
ouvrière française,
vous pouvez employer,
à temps de travail et
productivité équivalents,
9 Marocains, 25 Thaillandais,
35 Chinois, 65 Russes
ou 70 Vietnamiens." consulaires et économiques auprès du nouvel État. Bon nombre d'entre eux entretiennent avec leur pays d'origine des liens, dans la mesure où leurs familles sont restées en France. Depuis, leur nombre ne cesse de s'accroître en raison des investissements économiques français et de la présence également croissante des binationaux ou des expatriés. Les trois quarts de la population française du Maroc se concentrent à Casablanca et Rabat. Cette présence favorise la venue à Casablanca, considérée comme une "province française".

\section{S'associer avec un Marocain ou l'altérité entreprenariale}

Dans son acception première, "association" signifie participation à deux ou à plusieurs dans un projet ou dans une activité, dont les fruits et les profits seront partagés en fonction du taux de participation de chacune des parties. Cette définition économique et comptable dissimule un processus beaucoup plus complexe mettant en scène des convergences/divergences entre associés. Or, l'étude montre que $13 \%$ seulement des patrons enquêtés ont cité l'association avec un local comme un facteur incitatif, alors qu'ils sont très nombreux à passer par cette procédure. 


\section{Tableau V}

\begin{tabular}{|c|c|}
\hline Associés et non associés & Pourcentage (effectifs) \\
\hline Patrons ayant un associé marocain & $78(39)$ \\
\hline dont prête-nom & $26(13)$ \\
\hline association réelle & $52(26)$ \\
\hline Patron détenant $100 \%$ du capital de leur unité & $22(11)$ \\
\hline Total & $100(50)$ \\
\hline
\end{tabular}

On discerne l'existence de deux principales formes d'association. La première est fictive, dans la mesure où il s'agit de détourner la loi de la marocanisation ; la seconde est effective. La rationalité qui régit les rapports entre les deux associés n'est pas identique : l'associé français cherche à faire un investissement à long terme pour l'entreprise qu'il espère "récupérer" dans sa totalité. Quant à l'associé marocain, il cherche à vendre son savoir-faire local et à en tirer le profit maximum à très brève échéance. Cette complémentarité est, selon nous, porteuse d'une altérité qui se manifeste en termes d'emprunts, de négociations, de confiance et d'un échange approfondi. Pour élucider cette altérité, il convient de procéder à une lecture qui tranche les clivages entre l'universel et le particulier, le complexe et le simple, le rationnel et l'irrationnel, l'affectif et le bureaucratique. Les expériences relatées par nos interlocuteurs entendent faire la démonstration de la réversibilité des pratiques qu'induit l'association et du caractère mouvant des rapports entre associés. L'association est autant une contrainte qu'une stratégie. Le pragmatisme consiste à tirer parti du concret.

Ainsi, ceux pour qui s'allier à un patron local est une pure stratégie revendiquent-ils l'utilité de s'adapter aux réalités concrètes, "de ne rire ni de pleurer, mais de comprendre". L'associé est considéré comme un miroir qui donne à voir la société marocaine dans sa spécificité et sa profondeur : "Pour moi, ici à Casablanca et dans mes premières années au Maroc, mon associé était incontestablement un maître. Sa façon de composer face à des contraintes, et Dieu sait que nous en avons, des contraintes qui m'auraient moi déboussolé, cette façon est magistrale dans son genre. Il est incontestablement compétent à la marocaine, il anticipe les problèmes, sait parler à l'administration, les Marocains il les comprend mieux que quiconque, un inspecteur de travail qui réclame je ne sais quelle taxe, il le prend par l'épaule, lui chuchote quelques mots, des éclats de rire ct le tour est joué".

Certains patrons, constatant certaines pratiques de leur associé, se mettent à les décrier avant de les comprendre. Ainsi, un enquêté rapporte: "À peine nous sommesnous lancés dans la procédure d'association que mon associé, pourtant homme de raison et 
de rigueur, aborde la question des recrutements : il ne veut embaucher que ses cousins. Interpellé, je lui fais remarquer que s'agissant de cette question, on avisera le moment venu avec des critères précis et conjointement arrêtés et pour le bien de l'entreprise. Sèchement, il me rétorque que je ne connais pas les Marocains et qu'il vaut mieux travailler avec des individus que l'on connaît intimement et dont on connaît la famille, plutôt qu'avec des personnes triées sur un volet quelconque. Plus tard, je devais me convertir à sa théorie."

Cet extrait montrc à quel point l'altérité n'est pas synonyıne d'irrationalité. La rationalité n'existe pas en soi, elle est contenue dans le contexte social qui valorise certaines relations sociales, consacre certaines stratégies et honorent certains comportements. Au-delà de "l'incompatibilité" conjoncturelle des valeurs, l'interaction entre les deux associés obéit à la logique du donnant-donnant. Pour le partenaire français, la démarche d'association le dispense du casse-tête administratif. Dans l'ombre d'un associé local, c'est l'apprentissage de la vie marocaine qui est utilement entrepris : apprendre à connaître le milieu d'affaires, le fonctionnement socio-économique local d'une entreprise, les rapports avec les administrations et, par-dessus tout, la mentalité marocaine: "Mon associé n'a jamais manqué de me faire remarquer que c'est grâce à lui que je connais le Maroc. Il prend à chaque fois un plaisir à me réciter (et en arabe!) un hadith" qui dit que quand on se met à vivre avec un peuple pendant 40 jours, on devient l'un des leurs. Jai beaucoup appris dans son ombre..."

L'associé marocain gagnera en image auprès de la clientèle européenne dans ses stratégies futures. L'association est un art subtil et éphémère qui débouche souvent sur l'achat de l'entreprise par l'associé français.

Au terme de ces développements, on peut considérer que l'association révèle la pertinence de la culture de médiation dans le contact de la société marocaine et dans l'initiation à l'altérité pour le patron français. Elle souligne également la prégnance des face-à-face arrangés et bricolés pour les mettre en conformité avec l'intérêt économique. Les témoignages attestent de la densité du lien social à bâtir dans le sens de la convergence des intérêts et de la complémentarité des potentialités et des savoir-faire spécifiques.

\section{Atouts économiques et politiques}

C'est principalement au travers des facilités de séjours et de services que les patrons français élaborent leurs choix. L'accent est en effet porté sur les avantages de service qu'offre la société marocaine. Des types de rapports sociaux naissent ou émergent : ce sont des rapports de servitude. En règle générale, un patron français dispose d'une employée de maison, d'un gardien, d'un cuisinier, d'un chauffeur, d'un coursier ou 
d'une femme de ménage. Toutes ces personnes dépendent de lui. En choisissant les "avantages de service", le patron français s'aligne sur des pratiques dominantes dans la société marocaine. La "bonne" fait partie des figures les plus familières au Maroc. L'insuffisance, voire l'absence d'un État social garantissant la scolarisation des enfants en bas âge, l'inaccessibilité des établissements privés pour les ménages à revenu moyen, l'importance grandissante du travail féminin et la honte (l'incapacité culturelle) qui caractérise l'homme marocain réfractaire aux tâches domestiques ou ménagères constituent les causes de cette demande.

Par ailleurs, les patrons sont particulièrement sensibles à l'idée de la stabilité politique garantie par la monarchie chérifienne. Le roi est en effet considéré comme l'allié virtuel de la présence française au Maroc, tout comme on lui reconnaît la fonction de rempart contre le radicalisme religieux. Les patrons français louent aussi la culture du consensus national assurée par la monarchie.

\section{Au croisement entre plusieurs cadres de référence}

À l'aune des facteurs précédemment analysés, on peut dégager deux catégories qui ne sont pas étanches, mais complémentaires, tant il est admis que 'l'économique ne peut faire cavalier seul, le culturel est une branche du même arbre ${ }^{10}$. Les premières relèvent de l'universel ; les secondes se rapportent au singulier, c'est-à-dire au contexte sociétal marocain.

Au commencement, ce qui est ouvertement assumé par la totalité des patrons est cette stratégie de survie à laquelle ils sont acculés. Si les vagues de délocalisation varient, il demeure que la crise du secteur, les charges exorbitantes et l'action syndicale en France sont autant de facteurs à l'origine de la délocalisation. Parallèlement, l'option marocaine est motivée par différents coûts avantageux : ceux de la main-d'cuvre et du transport. Cette rationalité "instrumentale" anime en premier lieu tout entrepreneur établi dans un pays à "avantages comparatifs favorables". L'esprit d'entreprise est avant tout rationnel, on ne délocalise son activité que dans le but de réaliser d'abord du profit. La rationalisation illustre mieux les tendances profondes du régime moderne de la production. L'esprit d'entreprise repose également sur le calcul et sur la mesure. En cela, le projet économique tend à découvrir dans la rćalité du monde à conquérir tout ce qui pourrait donner lieu à la rentabilité économique (délocalisabilité). Dans la logique du patron, ce que nous avons appelé les "avantages de service" en terre marocaine représente des atouts économiques premiers : la "bonne", le loyer bon marché, le 
chauffeur, le cuisinier, le coût de la vie sont autant d'éléments qui concourent également à assurer le bien-vivre. Ces catégories ne relèvent pas de l'universel, elles ne sont pas pour autant typiquement marocaines.

Les mobiles culturels se rapportent à ce que Max Weber (1971) qualifié d'"axiologique", soit tout ce qui a trait aux valeurs, en s'inspirant d'une vision dégagée du calcul en termes de coût et de profit. Cette vision est imprégnée de perceptions et de représentations différentes selon les agents.

Les enquêtés ont cité l'existence d'une communauté française à Casablanca. Cette dernière est d'abord culturelle, symbole physique de l'entre-soi communautaire. Casablanca attire les délocalisations parce que l'essentiel de la communauté française y est concentré, contrairement à d'autres villes qui bénéficient d'une réputation dans le secteur textile, comme Fès ou Tanger. Cette communauté doit son existence, non à un quelconque décret officiel, - comme naguère en terre d'islam où les Dhimmis ${ }^{111}$ sont tolérés, mais séparés de la communauté des musulmans -, mais à un choix de vie dicté par le partage des valeurs communes et d'un mode de vie similaire. En contexte marocain, le patron français est un acteur social dont l'identité se construit en relation avec les valeurs marocaines, ou supposées telles.

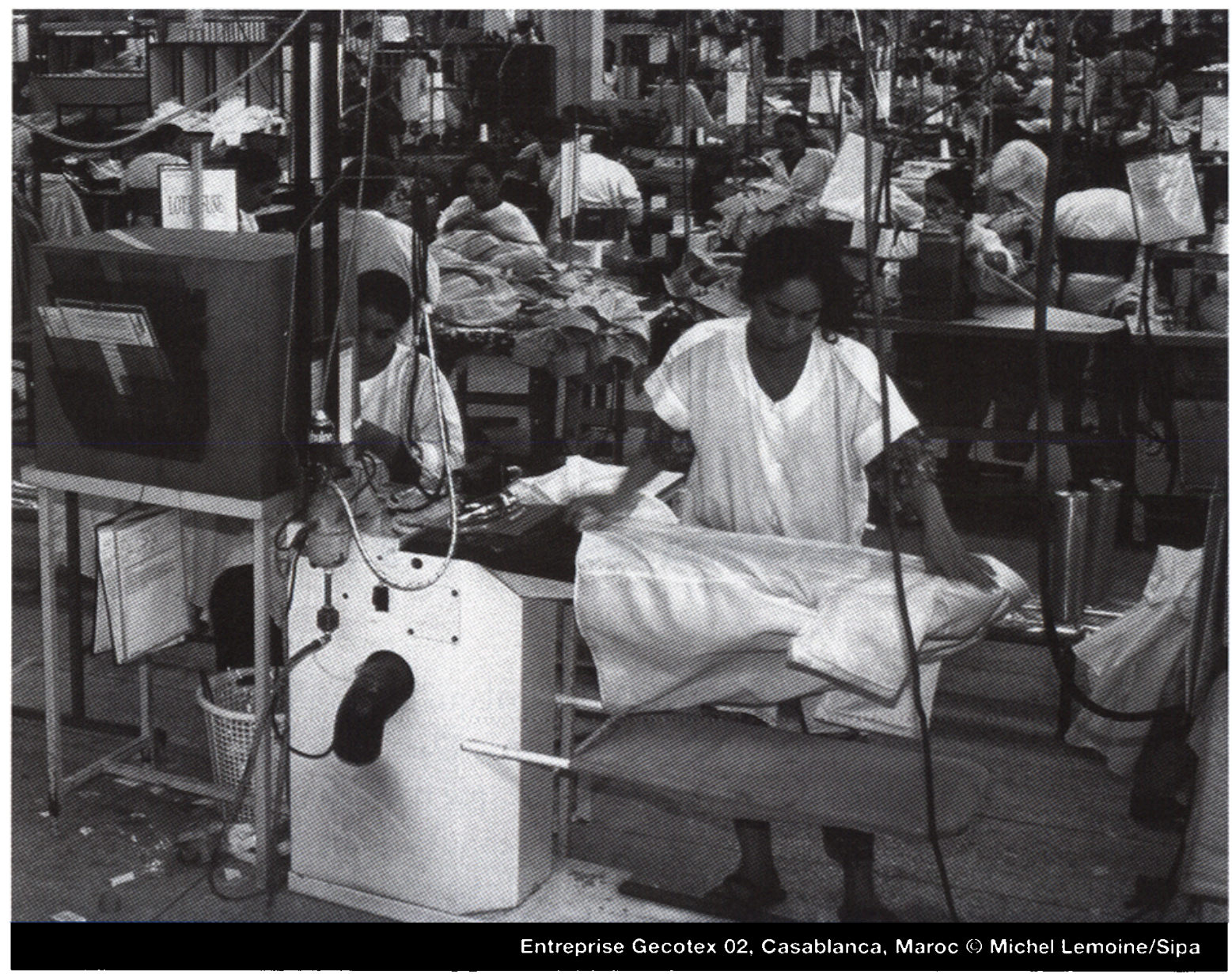


Sont loués le cadre de vie marocain, le soleil, la mer, la possibilité de s'adonner à des sports ou des loisirs tels que la pêche, la chasse ou le golf. Cette rationalité axiologique (par rapport aux valeurs) se rapporte en l'occurrence aux avantages extra-économiques dont le Maroc est censé regorger.

Cette pluralité des facteurs est en soi importante car elle permet justement de souligner la variété des catégories présidant à la délocalisation. Sans prendre en compte le degré d'autonomie juridique, nous avons retenu essentiellement un échantillon d'entreprises individuelles ou à capital mixte.

Les patrons enquêtés s'inscrivent dans une épaisseur sociale faite de contraintes, de ressources, de bricolages et de tout un rituel d'initiation. Ils sont détenteurs de certitudes leur conférant des connaissances exclusives et sans nuances sur le Maroc et les Marocains. Cette investigation renseigne sinon sur un paradoxe, du moins sur une dissonance : le Maroc attire, malgré les difficultés du secteur et le "retard" de l'administration à fournir des conditions favorables aux investisseurs. Ces derniers sont tenus à l'impératif de l'adaptation, nécessaire à la réussite entrepreneuriale. Cet impératif face à un milieu insaisissable et imprévisible est significatif de l'influence du contexte national dominant dans les stratégies des délocalisations. Nous pouvons dire de chaque patron qu'il est au carrefour de plusieurs cadres de références qui dictent sa conduite, aux prises avec le terrain local à conquérir.

Les témoignages renseignent en dernière analyse sur la centralité de l'enjeu économique, mais la justification de la délocalisation peut s'étendre à des dimensions "annexes" telles que le cadre de vie marocain et l'effet sociétal favorable à l'investissement. Malgré le credo d'un "Maroc compétitif" face à la concurrence, notamment de l'Europe de l'Est, certains patrons font de ces dimensions annexes le "balancier" qui les fait se maintenir au Maroc : "Il vaut mieux gagner moins et vivre mieux que le contraire." En conséquence, nous nous proposons de dégager une typologie de leurs rapports au Maroc.

\section{Deux types idéaux dans les patrons rencontrés}

Il n'y a pas de délocalisation sans l'acteur principal qui l'a décidé et mise en cuvre, face à des ressources mais aussi à des contraintes. Dans leur rapport au Maroc, deux types idéaux de patrons se détachent. Ceux que nous appelons les "néocoloniaux prudents" se caractérisent par une vision "condescendante" du Maroc et des Marocains, inspirée par un imaginaire colonial. Les "conquérants aventuriers" sont plus intéressés par les avantages que leur procure leur statut social. 
Les "néocoloniaux prudents", munis d'un projet détaillé ou ayant un associé local, sont à l'abri de toute aventure. La connaissance du marché marocain et des facilités d'implantation représentent leurs atouts premiers. Les seconds sont la figure la plus récente de ceux qui délocalisent. Ils ont bénéficié de l'abrogation de la loi de marocanisation qui les dispense du recours à un associé local, souvent "encombrant".

Les premiers appartiennent généralement à la première vague. Ils sont caractérisés par une méfiance politique et un savoir-faire économique. Les seconds ont cédé à la tentation du début des années quatre-vingt-dix avec une panoplie d'accords, et en particulier l'accord de l'association euroméditerranéenne.

Pour les "néocoloniaux prudents", la nécessité d'une implantation directe dans le pays hôte joue, au moins dans un premier temps, en faveur de l'association avec un partenaire local sous la forme d'une entreprise conjointe, plus communément appelée "joint-venture". On l'a souligné, cette formule vise à utiliser les ressources et les avantages offerts par les deux pays concernés ; elle est pratiquée prioritairement par les grandes entreprises à forte productivité. Pour le secteur textilehabillement, c'est plutôt une stratégie d'entreprise, assortie de quelques précautions pratiques. La première consiste à se mettre à l'abri de toute mésaventure : apprendre à connaître les contraintes marocaines pour mieux les esquiver par la suite. C'est l'associé marocain qui joue le rôle d'initiateur : il accomplit l'essentiel des démarches qui exigent un contact physique avec l'administration marocaine. En contrepartie et dans les comptes de l'implantation, est pris en compte le coût du bakchich(12), appelé aussi la "corruption indolore". Le travailleur marocain est appréhendé sous les traits d'un serviteur fidèle au travail. Cependant, les patrons relevant de ce type parlent ouvertement de la corruption de l'administration marocaine et de son caractère prébendier. Ils critiquent aussi la rigidité de la loi et son indétermination.

\section{Une vision conservatrice de la société marocaine}

Christian, directeur général d'une entreprise, impute cette rigidité à l'héritagc français au Maroc: "Le Maroc? Cest la complexité administrative héritée de la France, mise à la sauce des pays émergents. On a beau y parler français et évoluer dans un environnement législatif très inspiré de notre système, ce pays présente les caractéristiques communes à tous les États en voie de développement. ${ }^{113 ;}$ Ces procédés sont pratiqués par les entreprises d'autres secteurs : "J'ai essayé de travailler proprement, en misant sur la 
qualité de service et la rapidité d'intervention. Mais j'ai renoncé. Maintenant, je travaille à la marocaine, à coups de bakchich. Le pire, c'est que ça marche... Mon chiffre d'affaires du premier trimestre 2001 est déjà supérieur à celui de l'an dernier."

La logique du patron prudent est aussi de s'assurer de sa clientèle en présentant la délocalisation comme un bien commun. Une clientèle déjà constituée et fidélisée représente un gage important. En amont, et pour maintenir ce rapport de fidélité, est élaborée conjointement une sorte de deal : "Avant de partir, jai fait savoir mon projet à mes clients : ils s'étaient engagés à me rester fidèles moyennant un produit moins cher et de qualité. J'ai eu leur aval et c'est bon, ne serait-ce que psychologiquement." Ces patrons recherchent ainsi des soutiens avant de démarrer leurs unités dans des conditions prometteuses. Ils se refusent à considérer la délocalisation comme une politique présentant l'effet pervers de causer la ruine des entreprises autochtones ou à capital marocain majoritaire, en invoquant leur spécificité d'exporter exclusivement vers l'Europe avec une clientèle préalablement et certaine.

Par-delà la connaissance du marché marocain, les "néocoloniaux prudents" ont une appréhension conservatrice de la société marocaine. Méfiants, ils font valoir leur statut de résidents temporaires au Maroc. Signe de cette caractéristique : la plupart des patrons ne sont pas propriétaires de leur résidence. Ils en sont locataires et prêts à réagir à tout changement de situation politique ou économique. Les dépôts d'argent se font dans une banque française et non marocaine pour se mettre à l'abri de tout accident. Les banques françaises sont fortement présentes au Maroc, et la plupart des banques marocaines sont contrôlées par de grands groupes bancaires français ${ }^{(14)}$. Ils estiment être dans le collimateur de l'État qui réclame toujours et toujours plus : "Tout s'achète au Maroc, et on peut s'attendre à ce que tout s'écroule. On est sous une tente et on sait qu'on n'est pas éternel ici. Notre carte de séjour est seulement d'un an, renouvelable. L'État nous guette et nous persécute."

\section{Les "conquérants aventuriers" : la chute dans l'inconnu?}

Le conquérant aventurier est en quelque sorte un chercheur d'une industrie porteuse de profits immédiats. Il considère le capital détenu comme le "sésame de toutes les portes". Les mesures juridiques d'encouragement aux investisseurs nonnationaux accélèrent le départ de "petits patrons" vers le Maroc. Cette approche du marché et de l'implantation n'est pas précédée d'une démarche informatrice sur le fonctionnement de l'administration marocaine et sur la connaissance du fonctionnement sociopolitique local. 
Pour illustrer les limites de cette croyance, nous rapportons l'histoire de ce patron qui a délocalisé son unité en 1993 : "Un bon matin, le caïd vient me voir pour me demander en plein travail de faire un convoi de toutes les filles de l'atelier pour scander 'Vive le roi !' à l'occasion d'un passage royal. Premièrement, je lui ai expliqué que les filles ont un travail à finir ; deuxièmement, je lui ai fait comprendre que je ne partageais pas ces façons d'être ; troisièmement, j'ai refusé de laisser partir les filles pour une durée indéterminée. En règle générale, le convoi royal n'est jamais ponctuel... Elles peuvent attendre trois jours durant, et dans ce cas c'est la fermeture de l'entreprise assurée... Eh bien, on ne m'a jamais pardonné d'avoir dit non. On m'a fait payer cher ce comportement tout à fait normal. Impôts, tracasseries de tout genre, et jai été amené à plier bagages et à aller ailleurs."

L'une des situations observées révèle l'importance de la négociation, en tenant compte de l'effet sociétal marocain. Il s'agit du montage d'une tente dans la toiture de l'entreprise, destinée à abriter le surplus de tissus en attente d'utilisation. C'est une pratique tolérée dans les autres entreprises car ne requérant pas l'autorisation du wali (gouverneur). Étant "nouvel arrivant", ce patron a dû recevoir la visite de deux agents qui lui ont demandé de payer une amende pour avoir "illégalement" installé cette tente. Voulant justifier la légalité d'une telle pratique, d'échange en échange, il a refusé de s'acquitter de l'amende demandée. Cette décision lui a valu quelque temps plus tard une assignation à régler 9000 dirhams (environ 900 euros), faute de quoi il encourait la fermeture de l'entreprise. S'exécutant donc, il entreprend néanmoins un recours pour contester une mesure qu'il considère comme arbitraire: "J'ai dû prêcher dans le désert pendant un moment, jai finalement été reçu par un agent à la préfecture qui m'a dit crûment : 'Vous auriez dî composer avec ceux qui vous ont rendu visite!' Ici, c'est d'homme à homme que les problèmes se règlent. J'ai compris que le bakchich fait partie de notre existence."

La caractérisation des patrons français délocalisant leurs activités au Maroc fait ressortir un objet complexe que la littérature économique réduit à une simple équation économique. Si cet aspect est important, il n'est pas le seul, surtout dans un contexte marqué par des imaginaires croisés, héritage de tant d'années d'accumulation des connaissances parcellaires. Il est important de considérer cette dimension, à l'heure où les délocalisations industrielles sont essentiellement approchées sous l'angle des coûts et des avantages dans une économie mondialisée. 
Références bibliographiques

- Bernard Ch. (1983), "Une rationalité réappropriée par une autre ? Léconomique et le socioculturel dans la recherche sur le travail/développement", $A A N$, p. 56.5-582.

- Cassart Ch., Gérard M. (2001), "Concurrence salariale et délocalisations d'entreprises", revue Reflets et perspectives de la vie économique.

- Grange D. (1997), "La monarchie chérifienne, jeu de pouvoir et pouvoir du temps", Géopolitique n 57.

- Schroeter D. (1988), Merchants of Essaouira : Urban Society and Imperialism in Southwestern Morocco, 1844-1886, Cambridgc UP.

- Weber M. (1971), Économie et société. Paris, Plon.

- Zaim F. (1990). Le Muru et son espace méditerranéen : histoire économique er sociale, Rabat. Confluence.

\section{Notes}

1. Je remercie Marie Poinsot pour sa lecture attentive de la première version de ce texte.

2. Sauf mention contraire, les différentes citations sont celles des personnes enquêtées.

3. Schrocter, 1988 et Zaïn, 1990.

4. Labari. 2004.

5. Les avantages de service consistent à avoir une secrétaire, un chauffeur, un gardien et une employée de maison.

Il s'agit aussi de disposer aussi d'un capital social auprès des autorités locales.

6. Lancien roi Hassan II déclarc : "Le Maroc ressemble à un arbre dont les racines nourricières plongent profondément dans la terre d'Afrique, et qui respirc grâce à son feuillage au vent de l'Europe." (cité par Grange, 1997).

7. "Les investissements : état des lieux", Conjoncture, $1^{\circ} 821$, novembre 2001 . p. 14.

8. Cassart et Gérard, 2001,p. 35).

9. Paroles et actes du prophète rapportés par une chaine de témoins et consignés pour constituer, avec le Coran, la normativité en islam et source de la foi.

10. Bernard, 1983 , p. 323.

11. Danc la tradition chérifienne, les communautés non islamiques sont placées sous la protection directe du trône et constituent donc des $A h l$ Dhimma, statut accordé aux autres confessions dans le domaine de lisslam, à savoir la libcrté de culte. 12. Sclon la formulc populaire chez bon nombre de patrons, ce sont des "liquidités $51 \%$ ", référence au pourcentage que doit détenir une entreprise marocaine dans tout investissement ćtranger sous la loi de la marocanisation.

13. Cité par l'hebdomadaire L'Express, n' 2601, semaine du 10 au 16 mai 2001, p. 53.

14. Les banques françaises ont développé des partenariats avec les banques marocaines. C'est le cas du Crédit lyonnais avec le Crédit du Maroc, de la Banque nationale de Paris avec la BMCI (Banque marocaine du commerce et de lindustric), et de la Société générale avec la SGMB (Société générale marocaine des banques). 